\title{
Towards a Semiotic Communications Quality Model
}

\author{
Aldo de Moor and Hans Weigand \\ Infolab, Tilburg University, P.O.Box 90153, 5000 LE Tilburg, The Netherlands \\ tel.+31-13-4663020, fax+31-13-4663069, e-mail: \{ademoor|weigand\}@kub.nl
}

\begin{abstract}
The quality of communication processes in networked organizations is difficult to evaluate and improve, because of the many parties involved in meaning construction and responsibility assignment. This paper presents an outline of a communications quality model grounded in semiotics that can be used to construct a quality management system. Key elements of any such system are quality perspectives, processes, and attributes. To construct a semiotic communications quality model, we apply the quality elements to a semiotic communication process model. We then use Stamper's norm classificiation of perceptual, cognitive, evaluative, and behavioral norms to guide the various quality management processes.
\end{abstract}

Key words: Quality management, Information systems, Communication processes, Norms

\section{INTRODUCTION}

Information systems development has long been constrained to waterfalllike approaches aimed at producing large, transaction-based systems used for all kinds of computational and administrative purposes, such as payroll management, reservation systems, and so on (Brooks, 1995). The specification of those systems is quite straightforward: entities to represent in the databases and software programs are non-ambiguous and relatively easy to define, and responsibilities about who makes and owns the specifications are clear. However, information systems in the age of the Internet are much more communication than computation systems. They are a key part of the socio-technical system comprising the whole organization. Their applications to supporting complex communication processes, like discussion and group decision making, are manifold. In Table 1, we

The original version of this chapter was revised: The copyright line was incorrect. This has been corrected. The Erratum to this chapter is available at DOI: 10.1007/978-0-387-35611-2_22 
characterize some differences between information and communication systems.

Table 1. From Information to Communication Systems

\begin{tabular}{lll}
\hline & Information Systems & Communication Systems \\
\hline focus: & information & communication \\
supports: & transaction processes & communication processes \\
design objects: & clear specifications & "fuzzy" process-definitions \\
devt. process: & single project & continuous process \\
developers & elite development team & many stakeholders \\
\hline
\end{tabular}

Many have the uneasy intuition that such communication systems have great potential, which for some reason often fails to materialize, however. One main reason is that the semiotics of these systems are much more complex, particularly because the intended semantics and pragmatics are not under the control of one single actor, but negotiatable at best. In the resulting diffuse, volatile actor networks, the meaning of information produced and responsibilities for system use and specification are therefore often not clear.

In order to deal with such problems, we need to move away from the traditional information flow paradigm, in which positivistic modelling of symbol manipulating functions aimed at producing automated solutions is central. Instead, an information field paradigm is needed (Stamper, 2000). At the core of this paradigm are fields of norms, binding together groups of people. The norms allow meaning and responsibilities to be clearly specified, thus fostering the active construction of social reality, shared understanding and mutual commitments. The information systems built on the information field paradigm do not produce sterile data, but aim to generate and communicate information that can lead to true knowledge that helps people to perceive, understand, value, and act in the world. In Table 2, we summarize some differences between the respective paradigms, relevant for the design and use of information systems. We clearly see the information field paradigm to be better suited for the communication systems described. 
Table 2. From Information Flow to Information Field

\begin{tabular}{lll}
\hline & Information Flow-IS & Information Field-IS \\
\hline change: & static & dynamic \\
responsibility: & anonymous & individual responsibilities \\
design process: & representation & interpretation \\
objective: & control & perceive, understand, value, \\
& & act \\
control logic: & rules & norms \\
\hline
\end{tabular}

To better understand the role of norms in information systems, we distinguish between a deontic and a functionality space. The "deontic space" is defined by the norms and describes the acceptable operational behaviour of system users. It constrains the "functionality space", which is the total potential behaviour of users as enabled by the technical information system. It should be noted, however, that only some norms can be hard-wired into the technical options provided by the information technology, for example by allowing users with particular responsibilities access rights to certain files or functions. Still, many violations of the deontic space are possible, as often technical functionality allows for many different behaviours. To test and improve the quality of information systems in this sense, Stamper (2000) proposes meta-norms. In our interpretation, operational norms, forming the deontic space, guide the communication processes themselves, whereas the meta-norms guide their improvement through quality management processes.

In this paper, we investigate how such a quality management system could be constructed. We focus on the quality of communication processes, using examples from negotiation process support in a European B2B ecommerce project. In Sect.2, we examine the concept of quality as it is currently treated in the information systems literature. In Sect. 3, we outline a semiotic communications quality model consisting of a basic semiotic communication model and norm-governed quality management processes. Sect. 4 concludes the paper.

\section{QUALITY \& INFORMATION SYSTEMS DEVELOPMENT}

Now that much of the basic technological infrastructure such as PCs, software packages, and electronic networks have become widely available, the concept of quality is becoming increasingly important in the field. Comprehensive methods and philosophies like ISO9001 and Total Quality 
Management are used to standardize and certify information systems development practices, in order to improve their quality. However, such approaches, popular and useful as they may be, are no panaceas. They lead to much bureaucracy and many ill-understood documents, often do not end up in results that are directly useful for system developers, and do not deal with different perspectives and conflicts of interest (Braa, 1995). Moreover, such approaches are grounded in the information flow paradigm. Alternatively, a quality management approach grounded in the information field paradigm can help to optimize the information systems development process. Such an approach clarifies exactly who should be involved in which stage of the process and with what responsibility, thus leading to more involvement and better use of human expertise. We next distill some universal building blocks that should be present in any quality approach: quality perspectives, attributes, and processes. Systems, including information systems, consist of related entities. The boundaries and links between system elements can be conceptualized in many different ways. A perspective allows for a consistent demarcation of system building blocks. When studying the quality of systems, we want to say something of their structural and behavioural properties. The attributes are the relevant system properties seen from a certain perspective. Assessing the quality of a system consists of setting desired attribute values and comparing them with the actual values found in the system in operation. The setting, measuring, and evaluating of quality values is done in a set of related quality processes.

\subsection{Quality Perspectives}

There are many different perspectives on information systems quality, leading to different sets of quality processes and attributes. Many approaches are grounded in the software engineering tradition, and focus on optimizing technical quality. Others concentrate on improving use quality, focusing on how well applications fit the needs of individual users. However, not much attention has so far been paid to improving organizational (i.e. semiotic) quality (Braa, 1995).

\subsection{Quality Attributes}

Quality has both holistic and reductionistic aspects. On the one hand, quality is something that must be comprehensive, a system "has a good look and feel". However, for practical analysis and discussion purposes, more manageable quality constructs are needed. These constructs are called quality attributes. They describe aspects of the information system and its operational and development processes that are of relevance from the 
viewpoint of a certain quality domain. Examples of attributes are efficiency, integrity, and continuity, among many others. Single attributes can and should be the initial focus of attention. However, afterwards, there should always be a "common-sense" evaluation process to see if the results agree with the whole, intuitive picture. This is in line with the observation that tacit knowledge possessed by organizational subjects can never be completely formalized (Weigand and Dignum, 1997).

Quality attributes are either product or process attributes, as overall quality can only be accomplished when the quality is improved of both the outputs and the processes in which they are produced. Product attributes describe aspects of the deliverables or intermediate objects produced during system operations and development, whereas process attributes capture characteristics of these processes themselves. Furthermore, quality information is partially provided by the operational system, i.e. usage metrics, and is partially captured in the form of specific quality metainformation, such as results from interviews between auditors and users. To illustrate, the well known TAME software engineering quality approach distinguishes between quality information stored in its (operational) Software Engineering Models and the information stored in the (meta) Goal Question Metric Models (Oivo and Basili, 1992).

Quality attributes are often organized in quality trees. These organize the attributes in different dimensions that reflect the different perspectives on the information system. For example, one typical such tree, much used in Dutch systems development projects, is that of Delen and Rijsenbrij (1990). It organizes 41 attributes in four dimensions: the process dimension concerns the development of the information system, the static dimension the intrinsic aspects of the system and documentation, the dynamic dimension the operations of the working system, and the information dimension the information produced by the system as output.

\subsection{Quality Processes}

Quality improvement is not a one-time event, but a continuous organizational learning process. Quality management aims to define quality procedures and standards and checks that they are used. These management processes include quality assurance, quality planning, and quality control (Sommerville, 2001). Quality assurance entails the establishment of a framework of organizational quality procedures and standards, quality planning is their selection and adaptation for specific projects, while quality control makes sure that the selected procedures and standards are performed correctly. One important subprocess of quality control is quality 
measurement, in which the values of attributes are assessed, either by people or, sometimes, automatically.

When looking at this current state of affairs, what do we need to construct a true semiotic approach to communications quality management? First, our main interest should be the organizational perspective, focusing on how organizational communication can be improved. Second, useful quality attributes must be selected for optimizing organizational communication processes. Current attributes at most focus on the development of the information system and the qualities of the information per se (e.g. the process and information dimensions of Delen and Rijsenbrij), not on the role that this information plays in pragmatic communication processes. Third, we need to define practical quality management processes, including the definition, selection, and measurement of quality attributes.

\section{AN OUTLINE OF THE SEMIOTIC COMMUNICATIONS QUALITY MODEL}

To pay explicit attention to communication processes, we use a basic semiotic communication process model. To this model, we apply the quality perspectives, attributes, and processes discussed in the previous section. We then illustrate the use of Stamper's norm classification to guide the various quality management processes, so that responsibilities become clear.

\subsection{A Basic Semiotic Communication Process Model}

The communication process model that we adopt makes a distinction between three levels of abstraction in the communication process: the media level, the information level, and the communication level. The media level of communication describes the physical characteristics of the communication process. The question is: how? How are messages put across? The information level of communication has to with the data contents. It is not about how messages are transported, but which messages are transported. The communication level is about what people do with messages.

The model is similar to the distinction made in DEMO between the documentary level, the information level, and the essential level of messages (Dietz, 1994). Both models are grounded in the Language/Action Perspective (LAP) paradigm, which studies problems of organizations from the perspective of the conversations that are being conducted to get things done, and thus starts from a process view (see (Schoop and Taylor, 2001) for an overview of the state of the art in LAP research). In Table 3, we compare 
our LAP-model with Stamper's well-known semiotic ladder. The refinement offered by the ladder can be used very well to investigate a certain layer in more detail. For example, at the communication level, the goal-oriented aspects of communication should be investigated against the background of the organizational embedding of communication acts. These aspects relate to the pragmatic and social levels of the semiotic ladder, respectively.

Table 3. Relating LAP and OS-levels

\begin{tabular}{ll}
\hline Semiotic Ladder Levels & Basic Sem. Comm. Model Levels \\
\hline social & communication (organization) \\
pragmatic & communication (objective) \\
semantic & information (semantics) \\
syntactic & information (syntax) \\
empirical & media (dynamic aspects) \\
physical & media (structural aspects) \\
\hline
\end{tabular}

At each process model level, quality attributes can be provided. Quality attributes at the media level include media richness, interactivity, reliability and efficiency. Information quality attributes are for instance integrity, completeness, precision, and timeliness. Integrity constraints in the communication system can be used to enforce some of these qualities. An example of a complex communication level attribute is the communicative rationality expressed by communicating parties in their interactions.

Traditional quality management systems mainly focus on the lower two levels. In reaction to that, the Language/Action Perspective has emphasized the importance of the third level. A comprehensive quality management approach is thus needed that accounts for all levels and their dependencies.

A fundamental aspect of quality is fitness-for-use. The quality of a tool cannot be assessed without taking into account the goals it has to serve. As a consequence, total quality management should explicitly account for the dependencies between the levels. For example, communicative acts that are aimed at fixing commitments between parties are better served by a medium that offers persistence (such as paper or email), whereas explorative acts are sometimes better served by a medium that does not offer persistence (such as a face-to-face meeting or an untaped telephone call).

\subsection{Governing Communications Quality Management Processes with Norms}

The model should take an organizational semiotic perspective on information systems, including the three levels of the communication 
process model. The explicit attention given to the communication level distinguishes our model from perspectives focusing on the technical or use quality. For each layer, relevant quality attributes need to be selected. Then, for each attribute, a customized set of quality management processes needs to be defined.

Norms play a core role in a semiotic communications quality model in that they guide the quality management processes. The MEASUR approach provides us with an explicit operationalization and classification of norms (Stamper, 2000). All norms have the structure: IF condition THEN subject ADOPTS attitude TOWARD something. First, there are perceptual norms, which say how agents can identify entities in the world. Second, behavioral norms govern the actions of people, by making actions obliged, permitted, or forbidden. Third, cognitive norms represent who can have which beliefs (i.e. domain knowledge) about the world. Fourth, evaluative norms allow subjects to judge certain aspects.

Core to our approach is that for each combination of quality attribute and management process, a set of norms is defined. For example, take the quality control process of the "availability" attribute at the media level. A perceptual norm could say that a user can conclude that his mail inbox does not open anymore when a corresponding error message is received after starting the mail program. A cognitive norm could say that if a mail inbox does not open anymore, then the helpdesk expects that disk space is full. An evaluative norm can be used to conclude when the helpdesk thinks a mail service is faulty - for example, when the allocated disk space is less than 10 MB. Finally, behavioral norms represent the desired actions, for example, that the helpdesk should assign disk space for each new user within 1 day, or that users should clean up their mailbox when they receive a warning.

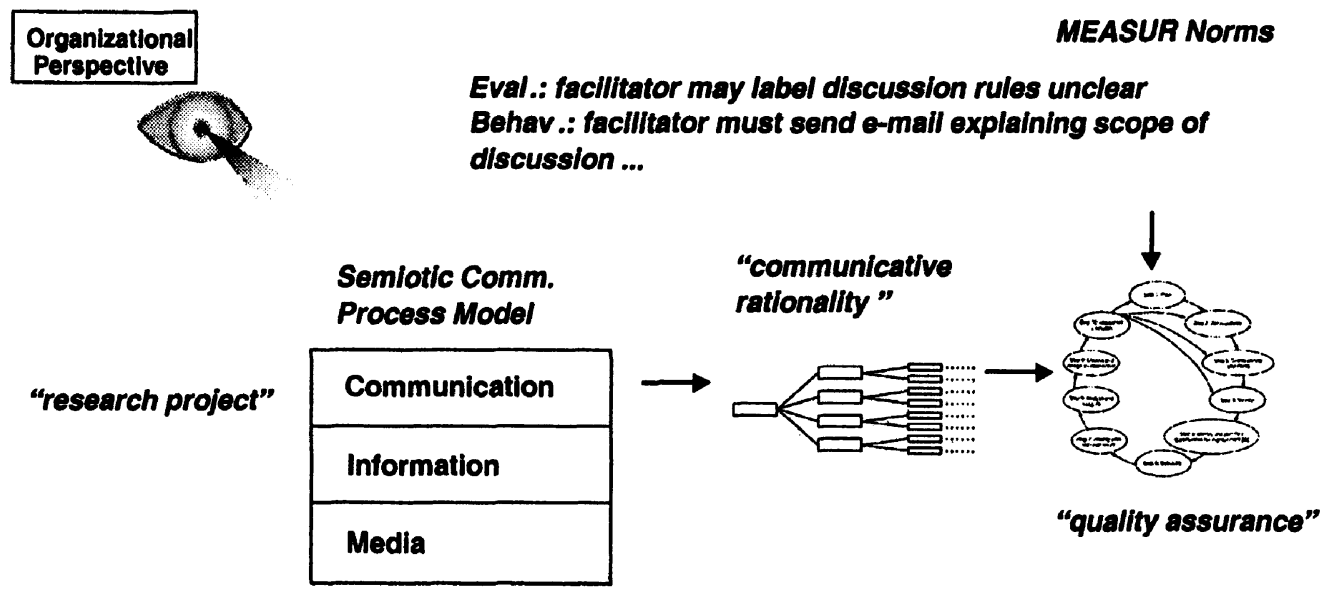

Figure 1. Applying the Semiotic Communications Quality Model 
Another example is the quality assurance process of the communicative rationality attribute at the communication process level (Fig.1). This has to do with the setting of the standards for this attribute, in other words, the definition of its desired value. One operational definition of communicative rationality is the kind of communication protocol applicable in a community, in other words: who can make what conversational moves in an interaction. Say that in an open research community normally everybody can raise and comment upon any kind of issue. However, whenever some participant thinks the discussion diverges too much, she may notify the discussion facilitator, who may in turn decide to freeze the current discussion, and explicitly define which topics are within the scope of the forum. Once finished, the open discussion can continue. An example of a perceptual norm here is that a discussion coordinator may see the number of complaints about a thread. A cognitive norm for the discussion facilitator could be that if two or more discussants complain about the discussion being confusing that the rules of engagement are likely not to be clear. An evaluative norm is that if the facilitator, upon asking the discussants about the reasons for their complaints, agrees that the discussion rules are not clear, then they are labelled "to be changed", with a certain degree of urgency, depending on the type of discussion. A behavioral norm would then be that if the rules of a discussion are to be changed, then the discussion facilitator is to send an email to all discussants summarizing the scope of the thread. In this way, the quality standards for the communicative rationality attribute of the discussion process are (re)set.

\subsection{Example: Improving the quality of a B2B Negotiation Process}

We apply the semiotic communication quality model to B2B negotiation, such as supported in the e-commerce MEMO project (see http://www.abnamro.com/memo). One of the negotiation protocols supported is a so-called tender-based negotiation protocol. This means that a buyer sends a request for bids to a open or closed set of potential sellers. The seller can reply using a bid message. This protocol is often used by contractors in the Dutch building sector.

The quality of the process can be managed at all three communication levels. The medium level quality is determined by attributes such as reliability of the medium (Internet vs. telephone) and timeliness. At the information level, the need for quality requires clarity of product identification terms. The use of standardized product identifications can contribute to this goal. Finally, at communication level, the protocol can be evaluated in the light of the organizational goals. One of the goals is to 
promote competition among sellers, to reduce prices and to comply with European laws. MEMO found that management sometimes complained about their purchasers not selecting enough potential sellers. One complex aspect that determines different attributes at the various levels is competitiveness. At the media level it may determine an attribute like security, which would entail that no company-specific files should be accessible by competing organizations. One - very specific - attribute at the communication process level could be competitor diversity, which would mean that enough companies bid for the tender. There are several norms involved, for example with respect to the quality control process of this attribute. First, the manager apparently has an evaluative norm of what is the appropriate number of potential suppliers to be involved in a tender (since he has the authority). This number can be fixed or depend on the amount or product category. To integrate the quality control process in the information system, and possible automate part of it, the manager should make this norm explicit. To improve the process, the manager can instruct the purchasers to increase the selection set - an example of a behavioral norm for the purchaser.

\section{CONCLUSIONS}

In this paper, we outlined the components of a quality management approach focused on the improvement of communication processes and grounded in organizational semiotics. Surveying the general quality of information systems literature, we identified quality perspectives, attributes, and processes as important elements of such an approach. To focus on the quality of communication processes, we started with a basic semiotic communication process model to which quality attributes are attached. The normative grounding of the related quality management processes was done by using the MEASUR classification of perceptual, cognitive, evaluative, and behavioral norms.

The novelty of this approach is its operationalization of general information systems quality theory in the organizational semiotics paradigm, as well as the explicit focus of quality management on communication processes, often neglected so far. Furthermore, we think it could be an interesting new application of the MEASUR methodology. Of course, in the limited space of this paper, we could only highlight some of the elements and applications of the methodology. In (Weigand and De Moor, 2001) we discuss the quality of communication processes in more depth. In future research, we intend to come up with a detailed typology of communication process attributes and the quality processes in which they are managed. 


\section{REFERENCES}

Braa, K. (1995) Beyond Formal Quality in Information Systems Design - A Framework for Information Systems Design from a Quality Perspective, in Proc. of the 18th IRIS Conference, Gjern, Denmark.

Brooks, F. (1995) The Mythical Man-Month: Essays on Software Engineering, AddisonWesley.

Delen, G.P.A.J. and Rijsenbrij, D.B.B. (1990) Quality Attributes of Automation Projects and Information Systems [transl.], Informatie, 32(1), pp. 46-55.

Dietz, J.L.G. (1994) Business Modelling for Business Redesign, in Proc. of the 27th HICSS Conference, IEEE Computer Press , pp.723-732.

Oivo, M. and Basili, V.R. (1992) Representing Software Engineering Models: the TAME Goal Oriented Approach, IEEE Trans. on Software Engineering, 18(10):886-898.

Schoop, M. and Taylor, J., eds. (2001). Proceedings of the Sixth International Workshop on the Language-Action Perspective on Communication Modelling, LAP 2001. July 2001, Montreal, Canada.

Sommerville, I. (2001) Quality Management, in Sommerville, I., Software Engineering, 6th ed., Addison-Wesley, pp.535-556.

Stamper, R. (2000) New Directions for Systems Analysis and Design, in Filipe, J. (ed.), Enterprise Information Systems, Kluwer Academic Publishers, London, pp.14-39.

Weigand, H. and Dignum, F. (1997) Formalization and Rationalization of Communication, in Proc. of the Second International Workshop on Communication Modelling, the Language/Action Perspective, Veldhoven, The Netherlands, June 9-10 1997, pp.71-86.

Weigand, H., and De Moor, A. (2001). A framework for the normative analysis of workflow loops, in Proc. of the Sixth International Workshop on Communication Modelling, the Language/Action Perspective, Montreal, Canada, July 2001, pp.31-50. 